\title{
Renormalization for the Laplacian and global well-possness of the Landau-Lifshitz-Gilbert equation in dimensions $n \geq 3$
}

Penghong Zhong ${ }^{1}$, Fengong $\mathrm{Wu}^{1 *}$ and Shengxiang Tang ${ }^{1}$

\section{"Correspondence:}

wufengong@gdei.edu.cn

'Department of Mathematics,

Guangdong University of

Education, Guangzhou, China

\section{Springer}

\begin{abstract}
The global solution of the $n \geq 3$ Landau-Lifshitz-Gilbert equation on $\mathbb{S}^{2}$ is studied under the cylindrical symmetric coordinates. An equivalent complex-valued equation in cylindrical symmetric coordinates is obtained by the Hasimoto transformation. A renormalization for the Laplacian is used to transform this equivalent system to a Ginzberg-Landau type system in which the Strichartz estimate can be applied. The global $H^{2}$ well-posedness of the Cauchy problem for the Landau-Lifshitz-Gilbert equation is established.
\end{abstract}

Keywords: Landau-Lifshitz equation; Well-posedness; Hasimoto transformation

\section{Introduction}

In this paper we consider the Cauchy problem for the Landau-Lifshitz-Gilbert (LLG) equation [14]:

$$
\frac{\partial}{\partial t} S=\alpha S \times \Delta S-\beta S \times(S \times \Delta S)
$$

where $S=\left(S_{1}, S_{2}, S_{3}\right), \alpha, \beta \in[0,1]$, the $\alpha$-term denotes the exchange interaction, while the $\beta$-term is the Gilbert damping term. Furthermore, we set $\alpha^{2}+\beta^{2}=1$ after a time-scaling.

Equation (1) is a mixture of two well-known partial differential equations: If $\beta=0,(1) \mathrm{de}-$ generates into the Schrödinger map equation; (1) also includes the well-known harmonic map heat flow under the setting of $\alpha=0$. There are a number of different definitions that are all equivalent to the Schrödinger map equation and the harmonic map heat flow under the smooth setting. The general formulation of the heat flow equation for harmonic maps into a Kähler manifold $(M, g(t))$ (domain manifold with an evolving metric $g(t)$ and $0 \leq t \leq T)$ is similar to the general formulation of the Schrödinger map equation. Let $\Gamma_{b, c}^{a}$ be the Christoffel symbol of the target manifold $\left(M, g\left(t_{0}\right)\right)$. The harmonic map heat flow $u:(M, g(t)) \mapsto\left(M, g\left(t_{0}\right)\right)$ is then described by

$$
\frac{\partial}{\partial t} S=\Delta_{g(t), g\left(t_{0}\right)} S,
$$

(c) The Author(s) 2020. This article is licensed under a Creative Commons Attribution 4.0 International License, which permits use, sharing, adaptation, distribution and reproduction in any medium or format, as long as you give appropriate credit to the original author(s) and the source, provide a link to the Creative Commons licence, and indicate if changes were made. The images or other third party material in this article are included in the article's Creative Commons licence, unless indicated otherwise in a credit line to the material. If material is not included in the article's Creative Commons licence and your intended use is not permitted by statutory regulation or exceeds the permitted use, you will need to obtain permission directly from the copyright holder. To view a copy of this licence, visit http://creativecommons.org/licenses/by/4.0/. 
where

$$
\Delta_{g(t), g\left(t_{0}\right)} S=\Delta_{g(t)} S+g^{i j}(x, t) \Gamma_{b, c}^{a}(S) \frac{\partial S^{b}}{\partial x^{i}} \frac{\partial S^{c}}{\partial x^{j}}
$$

in the local coordinates $x=\left(x_{1}, x_{2}, \ldots, x_{n}\right)$ of the domain manifold and in the same time the local coordinates $y^{a}$ of the target manifold.

Since the Kähler manifold $(M, g(t))$ has an action by a complex structure $J(S)$ in the tangent bundle, the Schrödinger map equation can be written

$$
\frac{\partial}{\partial t} S=J(S) \Delta_{g(t), g\left(t_{0}\right)} S
$$

In radial coordinates, we just discuss the flow on $\mathbb{S}^{2}$ which means $S_{1}^{2}+S_{2}^{2}+S_{3}^{2}=1$. In local coordinate around $S(t, r)$, we can define a complex transformation $z=\left(S_{1}-i S_{2}\right) /\left(1+S_{3}\right)$ in the complex plane with metric given by the expression

$$
g=\lambda(z, \bar{z}) d z d \bar{z}
$$

where $\lambda(z, \bar{z})$ is the conformal factor. If the target manifold is $\mathbb{S}^{2}$, we have

$$
\lambda(z, \bar{z})=\left(1+|z|^{2}\right)^{-2} .
$$

In terms of a conformal complex coordinate $z$ on $N$, where the metric is given by (2)-(3), (1) can be rewritten as

$$
i z_{t}-(\beta i-\alpha)\left(z_{r r}+\frac{n-1}{r} z_{r}+2(\ln \lambda)_{z} z_{r}^{2}\right)=0
$$

As we see, (1) transforms into a nonlinear Ginzburg-Landau type equation (4) with a derivative term. It will not be difficult to realize the transformation between $z$ and $S$. If $\Re(z)$ and $\Im(z)$ represent the real part and the imaginary part of the function $z$. Exactly, we can use the conversion

$$
\left(S_{1}, S_{2}, S_{3}\right)=\left(\frac{2 \Re(z)}{1+z \bar{z}}, \frac{2 \Im(z)}{1+z \bar{z}}, \frac{1-z \bar{z}}{1+z \bar{z}}\right)
$$

to realize this transformation.

Equation (4) is for the equations of motion in the stereographic representation except the south pole $P=(0,0,-1)$. If $\alpha=1,(4)$ is the Schrödinger map equation on $\mathbb{S}^{2}$. If $U_{0}$ denotes the Green function of the linear Schrödinger equation, the Duhamel formula indicates

$$
z^{ \pm}(t)=z(t)+\int_{t}^{ \pm \infty} U_{0}\left(t-t^{\prime}\right) \widetilde{F}\left(t^{\prime}\right) \mathrm{d} t^{\prime}
$$

where

$$
\widetilde{F}=2(\ln \lambda)_{z} z_{r}^{2}
$$


As is well known, the smallness condition always implies the existence of a global solution for the nonlinear Schrödinger equation. However, because there is a gradient nonlinear term $\nabla$ in (4) (also see (6)), the framework considered by Klainerman and Ponce [13] and Shatah [20] cannot be directly applied to the Schrödinger map system. In the initially almost parallel spins, Sulem, Sulem and Bardos [21] consider initial conditions such that $z$ is small data which initially components $S_{10}$ and $S_{20}$ are "small" and $S_{30}$ is close to 1 .

The Schrödinger map equation is the simplest and the most important part of the LLG equation. Up to now there are so many significant well-posedness results, especially the existence of global smooth solution (or weak solutions) to it. There is an extensive literature on the topic, which we do not attempt to survey. For a better understanding of the results and various techniques as regards the Schrödinger map equation, we refer the reader to $[2-4,8,12,18,19,21]$ (and the references therein).

Comparing to the Schrödinger map equation, the results of the well-posedness are even fewer. We just list some representative cases in dimension order below. If $n=1$ and with a periodic boundary condition setting, Guo and Huang [7] proved the existence of unique smooth solutions by means of the different methods. In dimension $n=2$, LLG equation is energy critical case which the $L^{2}$ norm for $\left|\nabla S_{\epsilon}\right|$ is conserved under the scaling $S_{\epsilon}(t, x)=S\left(t / \epsilon^{2}, x / \epsilon\right)$. Based on the Ginzburg-Landau approximations method, Harpes $[9,10]$ proved in two dimensions the global existence and uniqueness of weak solutions with at most finitely many singularities. In dimension $n \geq 3$, the LLG equation becomes super-critical with respect to the $L^{2}$ norm of $\left|\nabla S_{\epsilon}\right|$. In $n=3$, some necessary conditions for the existence of global weak solutions were presented by Alouges and Soyeur [1]. Melcher [16] proved the existence of partially regular weak solutions for it in dimension 3 . The existence of the time-periodic solution belonging to $C^{1}\left(\mathbb{R}, L^{2}\left(\Omega, \mathbb{R}^{3}\right)\right) \cap C\left(\mathbb{R}, H_{N}^{2}\left(\Omega, \mathbb{R}^{3}\right)\right)$ was proved by Huber [11] under the homogeneous Neumann boundary conditions. In dimension $n \geq 3$, Guo and Hong [6] proved a global existence theorem of solutions for the LLG equation from some $n$-dimensional manifold $M$ into the $\mathbb{S}^{2}$ based on the links between the harmonic maps and the solutions of the LLG equation. For a smallness initial condition, the global well-posedness on scaling invariant homogeneous Sobolev space (by Melcher [17]) and Morrey space (by Lin, Lai and Wang [15]) has been established in $n \geq 3$ by the moving frame technique. However, the singularity of the solution will develop from some special small initial data. For instance, Ding and Wang [5] proved that the solution of (1) will blow up in finite time in three and four dimensions under small special initial data. The exact solution is helpful to understand the property of the Schrödinger map equation (or the LLG equation) directly. We refer to [22-24] for more about how to construct the solution of these two equations.

In this paper we establish a relationship between the LLG equation and the nonlinear Ginzburg-Landau equation. We deal with a new complex system to prove the global existence and regularity for the LLG equation in $n \geq 3$ under some special conditions. Here we state our main theorem as follows.

Theorem 1.1 Assuming $n \geq 3$ and the initial data $S_{0} \in H_{\mathbb{R}^{n}}^{2}$ for the radial LLG equation

$$
S_{t}-\alpha S \times\left(S_{1 r r}+\frac{n-1}{r} S_{1 r}\right)+\beta S \times\left[S \times\left(S_{1 r r}+\frac{n-1}{r} S_{1 r}\right)\right]=0,
$$


$S_{0}$ satisfies $\left\|\partial_{x_{i}} \partial_{r} S_{0}\right\|_{L_{r}^{2}} \ll 1$ and $\left\|\partial_{r} S_{0}\right\|_{L_{r}^{2}} \ll 1(i=1,2, \ldots, n)$. Furthermore, assuming $p \geq 2$, $\frac{4 p}{3(p-2)}>2$ and $L^{2}\left(\mathbb{R}^{3}, \mu\right) \subset L^{2 p /(p-2)}\left(\mathbb{R}^{3}, \mu\right)$ are satisfied. Then for $(7)$ there exists a global solution in time:

$$
S \in C\left(\mathbb{R}, H_{\mathbb{R}^{n}}^{2}\right) \cap L_{\mathrm{loc}}^{p}\left(\mathbb{R}, W_{\mathbb{R}^{n}}^{2, p}\right) .
$$

At the same time, $\mathrm{Q}_{0} \in H_{\mathbb{R}^{n}}^{1}$ and the n-dimensional Ginzburg-Landau type equation

$$
\begin{aligned}
& i Q_{t}-(i \beta-\alpha)\left(Q_{r r}+\frac{(n-1) Q_{r}}{r}-\frac{(n-1) Q}{r^{2}}\right)+4 \beta Q \int_{0}^{r} \Im\left(\bar{Q} Q_{r}\right) \mathrm{d} r \\
& +2 \alpha\left(|Q|^{2} Q+2(n-1) Q \int_{0}^{r} \frac{|Q|^{2}}{r} \mathrm{~d} r\right)=0
\end{aligned}
$$

holds;

$$
\mathrm{Q} \in C\left(\mathbb{R}, H_{\mathbb{R}^{n}}^{1}\right) \cap L_{\mathrm{loc}}^{p}\left(\mathbb{R}, W_{\mathbb{R}^{n}}^{1, p}\right)
$$

\section{Remark 1.2}

For Theorem 1.1, we list some comments as follows.

1 If $n=2$ and $\beta=0$, (7) is the radial symmetric Schrödinger mapping equation studied by Chang, Shatah and Uhlenbeck [4]. Theorem 1.1 presents a global regularity result of the LLG equation in $n \geq 3$ dimensions. In addition, if $\beta=0$, Theorem 1.1 also indicates the solution of the $n \geq 3$ Schrödinger map equation is global under the smallness and embedded conditions.

2 Under the conditions $S_{0}-S_{\infty} \in H^{1} \cap W^{1, n}$ and $\left\|\nabla S_{0}\right\|_{L^{n}} \ll 1$, Melcher [17] obtains the global result of the LLG equation. Moreover, the space used by Melcher can be extended to the general Morrey space $M^{2,2}$. By an equivalent couple Ginzberg-Landau type equation, Lin, Lai and Wang [15] prove some similar global results on $M^{2,2}$ under the smallness condition $\nabla S_{0} \ll 1$. In both of their works $[15,17], S_{0}-S_{\infty} \in L^{2}\left(\mathbb{R}^{n}\right)$ is the necessary condition to ensure that the estimation method can be carried out on the whole domain. In Theorem 1.1, the constraint condition $S_{0}-S_{\infty}$ is removed.

The paper is written as follows. In Sect. 2, we establish some basic estimates of the Ginzburg-Landau semigroup. In Sect. 3, we deduce the equivalent complex GinzburgLandau type equations from the LLG equation. We also derive the renormalization for the Laplacian in the equivalent system. In Sect. 4, we prove the $n \geq 3$ global existence theorem for the equivalent equation and the LLG equation.

\section{The fundamental priori estimates}

The well-known fundamental estimate for the kernel of the Schrödinger equation can be extended to the Ginzburg-Landau equation. Note that the fundamental solution of the $n$-dimensional complex equation

$$
i Z_{t}-(i \beta-\alpha) \Delta Z=0
$$


is closely related to

$$
\mathbb{K}_{\alpha, \beta}(x, t)=[2 \pi(\beta+i \alpha) t]^{-\frac{n}{2}} e^{-\frac{|x|^{2}}{4(\beta+i \alpha) t}},
$$

which is a mixture of the Poisson kernel ( $\beta=0$ case) and the heat kernel ( $\alpha=0$ case).

It follows that

$$
\mathbb{K}_{\alpha, \beta}(x, t) \star \varphi=[2 \pi(\beta+i \alpha) t]^{-\frac{n}{2}} \int_{\mathbb{R}^{n}} e^{-\frac{|x-y|^{2}}{4(\beta+\alpha) t}} \varphi(y) \mathrm{d} y
$$

for all $t \neq 0$ and all $\varphi \in S\left(\mathbb{R}^{n}\right)$.

Equation (8) leads to a semigroup

$$
\left(U_{\alpha, \beta}(t) \varphi\right)(x)=\int_{R^{n}} \mathbb{K}_{\alpha, \beta}(x-y, t) \varphi(y) \mathrm{d} y .
$$

Furthermore, we denote

$$
\mathcal{A} \varphi(t)=\int_{0}^{t} U_{\alpha, \beta}\left(t-t^{\prime}\right) \varphi\left(t^{\prime}\right) \mathrm{d} t^{\prime}
$$

As is well known, in $n$ space dimensions, if $Z$ is the solution to the initial-boundary linear equation

$$
i Z_{t}-(i \beta-\alpha) \Delta Z=\psi, \quad Z(0)=\varphi,
$$

the Duhamel formula of (9) is as follows:

$$
Z=\left(U_{\alpha, \beta}(t) \varphi\right)(x)+\mathcal{A} \psi(t) .
$$

In order to estimate the $\left(U_{\alpha, \beta}(t) \varphi\right)(x)$ and $\mathcal{A} \psi(t)$, we need to develop some basic lemmas under the different norm spaces. Similar to the Schrödinger semigroup, the GinzburgLandau semigroup has a certain smoothing effect on the derivatives such as

$$
\left\|\left(U_{\alpha, \beta}(t) \varphi\right)(x)\right\|_{L_{x}^{p}} \leq\left\|\left(U_{1,0}(t) \varphi\right)(x)\right\|_{L_{x}^{p}} \lesssim|t|^{-\frac{n}{2}\left(1-\frac{2}{p}\right)}\|\varphi\|_{L_{x}^{p^{\prime}}},
$$

where $2 \leq p \leq \infty$ and $p^{\prime}$ with $\frac{1}{p}+\frac{1}{p^{\prime}}=1$.

According to (11), we obtain an estimate of the first component of (10) as follows.

Lemma 2.1 Let $2 \leq p \leq \infty$ and $\frac{1}{p}+\frac{1}{p^{\prime}}=1$. Then the dispersive inequality for the linear Ginzburg-Landau propagator is as follows:

$$
\left\|\left(U_{\alpha, \beta}(t) \varphi\right)(x)\right\|_{L_{x}^{p}} \lesssim|t|^{-\frac{n}{2}\left(1-\frac{2}{p}\right)}\|\varphi\|_{L_{x}^{p^{\prime}}}
$$

Remark 2.2 Lemma 2.1 applies to the extreme case $p=2$ and $p=\infty$ : the complex interpolation is

$$
\left\|\left(U_{1,0}(t) \varphi\right)(x)\right\|_{L_{x}^{2}}=\|\varphi\|_{L_{x}^{2}}
$$


while the dispersive estimate comes from

$$
\left\|\left(U_{1,0}(t) \varphi\right)(x)\right\|_{L_{x}^{\infty}} \lesssim|t|^{-n / 2}\|\varphi\|_{L_{x}^{1}}
$$

Remark 2.3 The same estimate holds with the norms of $L_{x}^{p}$ and $L_{x}^{p^{\prime}}$ replaced by the norms of $H^{s, p}\left(\mathbb{R}^{n}\right)$ and $H^{s, p^{\prime}}\left(\mathbb{R}^{n}\right)$ : If $t \neq 0$, then

$$
\left\|\left(U_{\alpha, \beta}(t) \varphi\right)(x)\right\|_{H^{s, p}} \leq(4 \pi|t|)^{-n\left(\frac{1}{2}-\frac{1}{\mathrm{p}}\right)}\|\varphi\|_{H^{s, p^{\prime}}} \quad \text { for all } \varphi \in S^{\prime}\left(\mathbb{R}^{n}\right),
$$

where $s \in \mathbb{R}$ and $2<p \leq \infty$.

Next, we present an estimate of the second component of (10). If we denote

$$
G(t)=\|\psi(t)\|_{L_{x}^{p^{\prime}}}
$$

and

$$
H(t)=C|t|^{-\frac{n}{2}\left(1-\frac{2}{p}\right)} .
$$

From the estimate (11), we obtain

$$
\|\mathcal{A} \psi(t)\|_{L_{x}^{p}} \lesssim \int_{0}^{t}\left|t-t^{\prime}\right|^{-\frac{n}{2}\left(1-\frac{2}{p}\right)}\left\|\psi\left(t^{\prime}\right)\right\|_{L_{x}^{p^{\prime}}} \mathrm{d} t^{\prime} \triangleq H * G .
$$

By the weak Young inequality and (12), we have

$$
\|H * G\|_{L_{t}^{q}} \lesssim\|H\|_{L_{t}^{\gamma}}\|G\|_{L_{t}^{q^{\prime}}}
$$

where

$$
\frac{1}{\gamma}=\frac{n}{2}\left(1-\frac{2}{p}\right), \quad \gamma>1 .
$$

We introduce the mixed space-time Lebesgue spaces norms as the set of all functions $\varphi$ with

$$
\|\psi\|_{L_{t}^{q} L_{x}^{p}} \triangleq\left(\int\left\|\psi\left(t^{\prime}\right)\right\|_{L_{x}^{p}}^{q} \mathrm{~d} t^{\prime}\right)^{\frac{1}{q}}
$$

Furthermore, we say that $(q, p)$ is a Strichartz pairs if

$$
\frac{2}{q}+\frac{n}{p}=\frac{n}{2}
$$

where $p$ and $q$ satisfy $2<q \leq \infty$ and $2 \leq p \leq \infty$.

According to (10), (13) indicates a control of the $\varphi=0$ case as

$$
\|Z\|_{L_{t}^{q} L_{x}^{p}} \lesssim\|\psi\|_{L_{t}^{q^{\prime}} L_{x}^{p^{\prime}}}
$$

Evidently, (15) gives an estimate of the $\mathcal{A} \psi(t)$ component as follows. 
Lemma 2.4 Let $(q, p)$ be the strict Strichartz pairs defined by (14). Then there exists a Hardy-Littlewood-Sobolev inequality as follows:

$$
\|\mathcal{A} \psi(t)\|_{L_{t}^{q} L_{x}^{p}} \lesssim\|\psi\|_{L_{t}^{q^{\prime}} L_{x}^{p^{\prime}}}
$$

More clearly, a combination of Lemmas 2.1-2.4 and the Duhamel formula (10) indicates the control of the norm of $Z$ which comes from (9). We claim the Strichartz estimate formula as follows.

Lemma 2.5 Let $(q, p)$ be the strict Strichartz pairs. In $n$ space dimensions, $Z$ is the solution of (9). Then $Z$ satisfies the following space-time Strichartz estimate:

$$
\|Z\|_{L_{t}^{\infty} L_{x}^{2}}+\|Z\|_{L_{t}^{q} L_{x}^{p}} \lesssim\|\varphi\|_{L_{x}^{2}}+\|\psi\|_{L_{t}^{q^{\prime}} L_{x}^{p^{\prime}}}
$$

As is well known, the dispersive inequality, Hardy-Littlewood-Sobolev inequality and space-time Strichartz estimate are very useful tools to prove the regularity of the Schrödinger equation. Similar to this situation, Lemmas 2.1-2.5 (especially the spacetime estimate Lemma 2.5) will be essential for solving the nonlinear Ginzburg-Landau equations.

\section{Equivalent equations and renormalization for the Laplacian}

\subsection{Equivalent equations}

It should be pointed out that for (4) as a nonlinear equation with a derivative term it is not easy to establish a global theorem. However, if we use the method of Hasimoto transformations, (1) can be represented by a complex equation, often without derivative component. Using this transformation, we have

$$
\Phi=\frac{\kappa}{2} \exp \left[i \int_{0}^{r} \tau(t, \tilde{r}) \mathrm{d} \tilde{r}\right]
$$

where

$$
\kappa=\left(S_{r} \cdot S_{r}\right)^{\frac{1}{2}} \quad \text { and } \quad \tau=\frac{S \cdot\left(S_{r} \times S_{r r}\right)}{\kappa^{2}}
$$

are the curvature and torsion in the Euclid space, respectively.

Noticing that if $S \in \mathbb{S}^{2}$, it is obvious that

$$
-S \times(S \times \Delta S)=\Delta S+|\nabla S|^{2} S
$$

because of $\vec{a} \times(\vec{b} \times \vec{c})=(\vec{a} \cdot \vec{c}) \vec{b}-(\vec{a} \cdot \vec{b}) \vec{c}$. We map $S$ on the unit tangent vector $e_{1}$ to obtain the time evolution equation of $e_{1}$ as follows:

$$
\frac{\partial}{\partial t} e_{1}-\alpha e_{1} \times \Delta e_{1}-\beta\left[\Delta e_{1}-\left(e_{1} \cdot \Delta e_{1}\right) e_{1}\right]=0,
$$

where

$$
\Delta e_{1}=e_{1 r r}+\frac{n-1}{r} e_{1 r} .
$$


If we set

$$
E=\left(e_{1}, e_{2}, e_{3}\right)^{T}
$$

the right hand coordinates relationship shows that

$$
E=\left(e_{2} \times e_{3}, e_{3} \times e_{1}, e_{1} \times e_{2}\right)^{T} .
$$

Meanwhile, the time derivative of $E$ satisfies

$$
\frac{\partial E}{\partial t}=A E
$$

where

$$
A=\left(\begin{array}{ccc}
0 & -\omega_{3} & \omega_{2} \\
\omega_{3} & 0 & -\omega_{1} \\
-\omega_{2} & \omega_{1} & 0
\end{array}\right) .
$$

By the Frenet formula of the moving frame method, the spatial derivatives of orthogonal basis $E$ can be represented as follows:

$$
\frac{\partial E}{\partial r}=B E
$$

where

$$
B=\left(\begin{array}{ccc}
0 & \kappa & 0 \\
-\kappa & 0 & \tau \\
0 & -\tau & 0
\end{array}\right) .
$$

Combining (17)-(21) with the compatibility condition

$$
\frac{\partial E}{\partial t \partial r}=\frac{\partial E}{\partial r \partial t}
$$

we map (7) onto a moving helical space curve in Euclid space. Finally, we obtain a complex system as follows:

$$
\begin{aligned}
& i \Phi_{t}-(i \beta-\alpha)\left(\Phi_{r r}+\frac{(n-1) \Phi_{r}}{r}-\frac{(n-1) \Phi}{r^{2}}\right)+4 \beta \Phi \int_{0}^{r} \Im\left(\bar{\Phi} \Phi_{r}\right) \mathrm{d} r \\
& +\alpha\left(2|\Phi|^{2} \Phi+4(n-1) \Phi \int_{0}^{r} \frac{|\Phi|^{2}}{r} \mathrm{~d} r\right)-\Phi R(t)=0,
\end{aligned}
$$

where $R(t)$ is a real-valued function,

$$
R(t)=\left(\int \tau_{t} \mathrm{~d} \tilde{r}\right)_{r=0} .
$$

Furthermore, if we use the transformation

$$
Q=\Phi \exp \left[-i \int_{0}^{t} R\left(t^{\prime}\right) \mathrm{d} \tilde{t^{\prime}}\right]
$$


(22) can be transformed into

$$
\begin{aligned}
& i Q_{t}-(i \beta-\alpha)\left(Q_{r r}+\frac{(n-1) Q_{r}}{r}-\frac{(n-1) Q}{r^{2}}\right)+4 \beta Q \int_{0}^{r} \Im\left(\bar{Q} Q_{r}\right) \mathrm{d} r \\
& \quad+\alpha\left(2|Q|^{2} Q+4(n-1) Q \int_{0}^{r} \frac{|Q|^{2}}{r} \mathrm{~d} r\right)=0 .
\end{aligned}
$$

\subsection{Renormalization for the Laplacian}

Neither (22) nor (23) is a standard Ginzberg-Landau system due to the pseudo-Laplacian as follows:

$$
L(n, Q)=Q_{r r}+\frac{(n-1) Q_{r}}{r}-\frac{(n-1) Q}{r^{2}}
$$

If we do not transform (23) into a standard complex equation with a Laplacian, it is difficult to employ the Strichartz estimate. Under the setting of $n \geq 3$, the rearranging need two steps from $n=3$. Hence, we firstly reform the $L(n, Q)$ into a Laplace operator for the $n=3$ case.

In this case, (24) will become a Laplace operator under some special transformation. For the 3-dimensional case, we look for a variable separation transformation $U=Q(t, r) Y$ to rewrite (22) as

$$
i U_{t}-(i \beta-\alpha) \Delta U=H(Q, Y)
$$

where $Y$ is a function to be determined.

In spherical coordinates $(r, \theta, \varphi)(\varphi \in[0,2 \pi]$ and $\theta \in[0, \pi])$, we construct $U$ in the form of $U=Q(t, r) Y(\theta, \varphi)$. Hence, the Laplace operator is

$$
\Delta U=(\Delta Q) Y+2((\nabla Q) \cdot(\nabla Y))+Q(\Delta Y)
$$

If $Y$ is some spherical harmonic, which is a special function defined on the surface of a sphere (denoted as $Y_{\ell}^{m}(\theta, \varphi)$ or $\left.Y_{\ell}^{m}\right)$, we have

$$
(\nabla Q) \cdot\left(\nabla Y_{\ell}^{m}(\theta, \varphi)\right)=0
$$

and

$$
\Delta Y_{\ell}^{m}(\theta, \phi)=-\frac{\ell(\ell+1)}{r^{2}} Y_{\ell}^{m}(\theta, \varphi)
$$

More clearly, if we set

$$
Y_{1}^{\mp 1}= \pm \frac{1}{2} \sqrt{\frac{3}{2 \pi}} \sin \theta e^{\mp i \varphi},
$$

then the Laplace of

$$
U=Q Y_{1}^{\mp 1}
$$




$$
\Delta U=L(2, Q) Y_{1}^{\mp 1}
$$

By Laplace's spherical harmonics $Y_{1}^{1}$, we use the transformation $M=Q Y_{1}^{1}$ (or $Q=$ $\left.\left(Y_{1}^{1}\right)^{-1} M\right)$ to rewrite (23) as follows:

$$
i M_{t}-(i \beta-\alpha) \Delta M=H_{3}
$$

where

$$
\Delta M=\frac{1}{r^{2}} \frac{\partial}{\partial r}\left(r^{2} \frac{\partial M}{\partial r}\right)+\frac{1}{r^{2} \sin \theta} \frac{\partial}{\partial \theta}\left(\sin \theta \frac{\partial M}{\partial \theta}\right)+\frac{1}{r^{2} \sin ^{2} \theta} \frac{\partial^{2} M}{\partial \varphi^{2}}
$$

and

$$
H_{3}=-4 \beta M \int_{0}^{r} \Im\left(\bar{Q} Q_{r}\right) \mathrm{d} r-\alpha\left(2 M|Q|^{2}+8 M \int_{0}^{r} \frac{|Q|^{2}}{r} \mathrm{~d} r\right) .
$$

Now we finish the rearranging process for the $n=3$ case. The second step for the general $n$ is the case $n \geq 4$ which will be finished below. If $n \geq 4$, the Laplacian in more than four dimensions (for the Euclidean Laplacian in spherical polar coordinates) decomposes as

$$
\Delta=r^{1-n} \frac{\partial}{\partial r}\left(r^{n-1} \frac{\partial}{\partial r}\right)+r^{-2} \Delta_{S^{n-1}}
$$

where $\Delta_{S^{n-1}}$ is the Laplace-Beltrami operator on $S^{n-1}$. This operator is the analog of the angular part of the Laplacian in $n=3$. The spherical Laplacian is defined by

$$
\Delta_{S^{n-1}} Y(x)=\Delta Y\left(\frac{x}{|x|}\right)
$$

where $f(x /|x|)$ is the degree zero homogeneous extension of the function $\mathrm{f}$ to $\mathbb{R}^{n}-0$. An orthogonal basis of spherical harmonics in higher dimensions can be constructed inductively by the method of separation of variables, by solving the Sturm-Liouville problem for the spherical Laplacian

$$
\Delta_{S^{n-1}} Y\left(\theta_{2}, \theta_{1}\right)=\sin ^{2-n} \theta_{1} \frac{\partial}{\partial \theta_{1}}\left(\sin ^{n-2} \theta_{1} \frac{\partial}{\partial \theta_{1}}\right)+\sin ^{-2} \theta_{1} \Delta_{S^{n-2}} Y\left(\theta_{3}, \theta_{2}\right) .
$$

In particular, for the ordinary 2-sphere using standard notation for polar coordinates we have

$$
\nabla_{S^{2}}^{2} Y(\theta, \phi)=(\sin \phi)^{-1} \frac{\partial}{\partial \phi}\left(\sin \phi \frac{\partial U}{\partial \phi}\right)+(\sin \phi)^{-2} \frac{\partial^{2}}{\partial \theta^{2}} U .
$$

Now, we want to find a function which rearranges (24) into a Laplacian. According to (27) and (28), we need to construct a function (for the $n>3$ case) which satisfies

$$
\Delta_{S^{n-1}} Y=-(n-2) Y .
$$


The spherical harmonics which is on the unit sphere $S^{2}$ inside the three-dimensional Euclidean space can be generalized to the higher-dimensional Euclidean space. Furthermore, these are the solid spherical harmonics $Y\left(\theta_{n}, \ldots, \theta_{1}\right)$ on the space of functions on the unit sphere satisfying

$$
\Delta_{S^{n-1}} Y\left(\theta_{1}, \ldots, \theta_{n-1}\right)=-(n-2) Y\left(\theta_{1}, \ldots, \theta_{n-1}\right) .
$$

The end result of such procedure (29)-(30) generates the solution of (31):

$$
Y_{l_{1}, \ldots, l_{n-1}}\left(\theta_{1}, \ldots, \theta_{n-1}\right)=\frac{1}{\sqrt{2 \pi}} e^{i l_{1} \theta_{1}} \prod_{j=2}^{n-1}{ }_{j} \bar{P}_{l_{j}}^{l_{j-1}}\left(\theta_{j}\right)
$$

where the indices satisfy $\ell_{1} \leq \ell_{2} \leq \cdots \leq \ell_{n-1}$. The function ${ }_{j} \bar{P}_{l_{j}}^{l_{j-1}}\left(\theta_{j}\right)$ in (32) is defined in terms of the Legendre function $(\theta \in[0, \pi])$, thus

$$
{ }_{j} \bar{P}_{L}^{l}(\theta)=\sqrt{\frac{2 L+j-1}{2} \frac{(L+l+j-2) !}{(L-l) !}} \sin ^{\frac{2-j}{2}}(\theta) P_{L+\frac{j-2}{2}}^{-\left(l+\frac{j-2}{2}\right)}(\cos \theta)
$$

where

$$
P_{\ell}^{m}(\cos \theta)=(-1)^{m}(\sin \theta)^{m} \frac{d^{m}}{d(\cos \theta)^{m}}\left(P_{\ell}(\cos \theta)\right)
$$

and $P_{\ell}(y)$ is given by the Rodrigues formula,

$$
P_{\ell}(y)=\frac{1}{2^{\ell} \ell !} \frac{d^{\ell}}{d y^{\ell}}\left[\left(y^{2}-1\right)^{\ell}\right]
$$

The functions described by (33) satisfy the general Legendre differential equation with the indicated values of the parameters $\ell$ as follows:

$$
\left(1-y^{2}\right) \frac{d^{2}}{d y^{2}} P_{\ell}(y)-2 x \frac{d}{d y} P_{\ell}(y)+\ell(\ell+1) P_{\ell}(y)=0
$$

More clearly, we can list some polynomials given by (33) and (34) as follows:

$$
\begin{aligned}
& P_{1}^{0}(\cos \theta)=\cos \theta, \quad P_{1}^{1}(\cos \theta)=-\sin \theta, \\
& P_{2}^{0}(\cos \theta)=\frac{1}{2}\left(3 \cos ^{2} \theta-1\right), \quad P_{2}^{1}(\cos \theta)=-3 \cos \theta \sin \theta, \\
& P_{2}^{2}(\cos \theta)=3 \sin ^{2} \theta, \quad \ldots .
\end{aligned}
$$

Hence, (33) and (34) leave us with $P_{\ell}^{m}(\cos \theta)$ (hence ${ }_{j} \bar{P}_{L}^{l}(\theta)$ ) is a polynomial generated by $\sin \theta$ and $\cos \theta$. We can use the transformation $U=Q Y\left(Y\right.$ is short for $\left.Y\left(\theta_{n-1}, \ldots, \theta_{1}\right)\right)$ to rewrite (24) into a Laplacian. At the same time, we can transform (23) into

$$
i U_{t}-(i \beta-\alpha) \Delta U=H_{n}(Q, Y),
$$


where

$$
\Delta U=r^{1-n} \frac{\partial}{\partial r}\left(r^{n-1} \frac{\partial}{\partial r} U\right)+r^{-2} \Delta_{S^{n-1}} U
$$

and

$$
H_{n}=-4 \beta U \int_{0}^{r} \Im\left(\bar{Q} Q_{r}\right) \mathrm{d} r-\alpha\left(2 U|Q|^{2}+4(n-1) U \int_{0}^{r} \frac{|Q|^{2}}{r} \mathrm{~d} r\right) .
$$

Differentiating (with respect to $\left.x_{j}, j=1,2, \ldots, n\right)(35)$ we get

$$
i U_{x_{j} t}-(i \beta-\alpha) \Delta U_{x_{j}}=\widetilde{H}_{n},
$$

where

$$
\begin{aligned}
\left|\widetilde{H_{n}}\right| \lesssim & \frac{|U||Q|^{2}}{r}+|U| \Re\left(Q_{x_{i}} \bar{Q}\right) \\
& +\left|U_{x_{j}}\right|\left(|Q|^{2}+\int_{0}^{r} \Im\left(\bar{Q} Q_{r}\right) \mathrm{d} r+\int_{0}^{r} \frac{|Q|^{2}}{r^{\prime}} \mathrm{d} r^{\prime}\right) \\
\lesssim & \frac{|Q|^{3}}{r}+|Q| \Re\left(Q_{x_{i}} \bar{Q}\right) \\
& +\left|U_{x_{j}}\right|\left(|Q|^{2}+\int_{0}^{r} \Im\left(\bar{Q} Q_{r}\right) \mathrm{d} r+\int_{0}^{r} \frac{|Q|^{2}}{r^{\prime}} \mathrm{d} r^{\prime}\right) .
\end{aligned}
$$

The spherical coordinate system defined for the three-dimensional Euclidean space can be extend to the $n$ dimensional Euclidean space, in which the coordinates consist of a radial coordinate $r$ and $n-1$ angular coordinates $\theta_{1}, \theta_{2}, \ldots, \theta_{n-1}$ where the angles $\theta_{2}, \theta_{3}, \ldots, \theta_{n-1}$ range over $[0, \pi]$ and $\theta_{1}$ ranges over $[0,2 \pi]$. If $x_{i}$ is for the Cartesian coordinates, then we may compute $x_{1}, \ldots, x_{n}$ with

$$
\begin{aligned}
& x_{1}=r \cos \left(\theta_{n-1}\right), \quad x_{2}=r \sin \left(\theta_{n-1}\right) \cos \left(\theta_{n-2}\right), \\
& x_{3}=r \sin \left(\theta_{n-1}\right) \sin \left(\theta_{n-2}\right) \cos \left(\theta_{n-3}\right), \quad \ldots, \\
& x_{n-1}=r \sin \left(\theta_{n-1}\right) \cdots \sin \left(\theta_{2}\right) \cos \left(\theta_{1}\right), \\
& x_{n}=r \sin \left(\theta_{n-1}\right) \cdots \sin \left(\theta_{2}\right) \sin \left(\theta_{1}\right) .
\end{aligned}
$$

The volume element in the $n$ dimensional case in radial coordinates $\left(r, \theta_{1}, \theta_{2}, \ldots, \theta_{n-1}\right)$ is calculated from the Jacobian of the transformation

$$
\frac{\partial\left(x_{i}\right)}{\partial\left(r, \theta_{j}\right)}=\left(\begin{array}{cccccc}
\mathbf{C}_{n-1} & -r \mathbf{S}_{n-1} & 0 & 0 & \cdots & 0 \\
\mathbf{S}_{n-1} \mathbf{C}_{n-2} & r \mathbf{C}_{1} \mathbf{C}_{n-2} & -r \mathbf{S}_{n-1} \mathbf{S}_{n-2} & 0 & \cdots & 0 \\
\vdots & \vdots & \vdots & \ddots & \vdots \\
& & & & & 0 \\
\mathbf{S}_{n-1} \ldots \mathbf{S}_{2} \mathbf{C}_{1} & \cdots & \cdots & & & -r \mathbf{S}_{n-1} \cdots \mathbf{S}_{2} \mathbf{S}_{1} \\
\mathbf{S}_{n-1} \ldots \mathbf{S}_{2} \mathbf{S}_{1} & r \mathbf{C}_{n-1} \cdots \mathbf{S}_{1} & \cdots & & & r \mathbf{S}_{n-1} \cdots \mathbf{S}_{2} \mathbf{C}_{1}
\end{array}\right)
$$

where $\mathbf{S}_{i}$ and $\mathbf{C}_{i}(i=1, \ldots, n-1)$ denote $\sin \left(\theta_{i}\right)$ and $\cos \left(\theta_{i}\right)$, respectively. 
Precisely, the volume element is

$$
\begin{aligned}
d^{n} \mathcal{V} & =\left|\operatorname{det} \frac{\partial\left(x_{i}\right)}{\partial\left(r, \theta_{j}\right)}\right| d r d \theta_{1} d \theta_{2} \cdots d \theta_{n-1} \\
& =r^{n-1} \sin ^{n-2}\left(\theta_{n-1}\right) \sin ^{n-3}\left(\theta_{n-2}\right) \cdots \sin \left(\theta_{2}\right) d r d \theta_{1} d \theta_{2} \cdots d \theta_{n-1}
\end{aligned}
$$

Hence, the volume of the $n$-ball is calculated by

$$
\mathcal{V}_{n}=\int_{\theta_{1}=0}^{2 \pi} \int_{\theta_{2}=0}^{\pi} \cdots \int_{\theta_{n-1}=0}^{\pi} \int_{r=0}^{R} d^{n} \mathcal{V} \triangleq \int_{\left(\theta_{1}, \theta_{2}, \ldots, \theta_{n-1}\right)} \int_{r=0}^{R} d^{n} \mathcal{V}
$$

This indicates the $L^{2}$ norm of $U$ on the $n$-spherical coordinates is as follows:

$$
\begin{aligned}
\|U\|_{L_{\mathbb{R}^{n}}^{2}}^{2}= & \|Q Y\|_{L_{\left(r, \theta_{1}, \theta_{2}, \ldots, \theta_{n-1}\right)}^{2}}^{2}|Y|^{2} \sin ^{n-2}\left(\theta_{n-1}\right) \sin ^{n-3}\left(\theta_{n-2}\right) \cdots \sin \left(\theta_{2}\right) d \theta_{1} d \theta_{2} \cdots d \theta_{n-1} \\
= & \int_{\left(\theta_{1}, \theta_{2}, \ldots, \theta_{n-1}\right)} \\
& \cdot \int_{r}|Q|^{2} r^{n-1} \mathrm{~d} r \\
\lesssim & \|Q(t)\|_{L_{r}^{2} .}^{2}
\end{aligned}
$$

\section{Global solution in $n \geq 3$}

\subsection{Global solution in $n=3$}

We multiply (25) by $\bar{M}$, perform the integral and take the imaginary part. We have

$$
\frac{1}{2}\|M(t)\|_{L_{\mathbb{R}^{3}}^{2}}^{2}-\frac{1}{2}\left\|M_{0}\right\|_{L_{\mathbb{R}^{3}}^{2}}^{2}+\beta \int_{\mathbb{R}^{3}}\|\nabla M(\tau)\|_{L_{\mathbb{R}^{3}}^{2}}^{2} \mathrm{~d} \tau=0
$$

From (37), the norm of $M$ on spherical coordinates $(r, \theta, \varphi)$ can be controlled by

$$
\|M(t)\|_{L_{\mathbb{R}^{3}}^{2}}^{2} \leq\left\|M_{0}\right\|_{L_{\mathbb{R}^{3}}^{2}}^{2}
$$

According to $M=Q Y_{1}^{1},(38)$ also indicates a control of the norm on variable $r$

$$
\|Q(t)\|_{L_{r}^{2}}^{2} \leq\left\|Q_{0}\right\|_{L_{r}^{2}}^{2}
$$

It is obvious that

$$
\|M\|_{L_{\mathbb{R}^{3}}^{2}}^{2}=\left\|Q Y_{1}^{1}\right\|_{L_{(r, \theta, \varphi)}^{2}}^{2}=\int_{(\theta, \varphi)}\left|Y_{1}^{1} \sin \theta\right|^{2} \mathrm{~d} \theta \mathrm{d} \varphi \int_{r}|Q|^{2} r^{2} \mathrm{~d} r \lesssim\|Q(t)\|_{L_{r}^{2}}^{2} .
$$

By Lemma 2.5, we have

$$
\|M\|_{L^{\infty} L_{\mathbb{R}^{3}}^{2}}+\|M\|_{L_{\mathbb{R}^{3}}^{p}} \lesssim\left\|M_{0}\right\|_{L_{\mathbb{R}^{3}}^{2}}+\|H\|_{L_{\mathbb{R}^{3}}^{p /(p-1)}}
$$


According to $\|M\|_{L_{\mathbb{R}^{3}}^{2}} \lesssim\|Q\|_{L_{r}^{2}}$, (26) and (40), we obtain

$$
\begin{aligned}
\|M\|_{L_{\mathbb{R}^{3}}^{p}} \lesssim & \left\|Q_{0}\right\|_{L_{r}^{2}}+\left\|M|Q|^{2}\right\|_{L_{\mathbb{R}^{3}}^{p /(p-1)}}+\left\|M \int_{0}^{r} \frac{|Q|^{2}}{r} \mathrm{~d} r\right\|_{L_{\mathbb{R}^{3}}^{p /(p-1)}} \\
& +\left\|M \int_{0}^{r} \Im\left(\bar{Q} Q_{r}\right) \mathrm{d} r\right\|_{L_{\mathbb{R}^{3}}^{p /(p-1)}} \\
\lesssim & \left\|Q_{0}\right\|_{L_{r}^{2}}+\|Q\|_{L_{r}^{2 p /(p-2)}}^{2}\|Q\|_{L_{r}^{p}}+\left\|Q \int_{0}^{r} \frac{|Q|^{2}}{r} \mathrm{~d} r\right\|_{L_{r}^{p /(p-1)}} \\
& +\left\|Q \int_{0}^{r} \Im\left(\bar{Q} Q_{r}\right) \mathrm{d} r\right\|_{L_{r}^{p /(p-1)}} .
\end{aligned}
$$

We notice that the estimate of $Q \int_{0}^{r} \Im\left(\bar{Q} Q_{r}\right) \mathrm{d} r$ is similar to $|Q|^{2} Q$. By the Young inequality and the Hardy inequality, we obtain the estimate of the last two terms of (41) as follows:

$$
\left\|Q \int_{0}^{r} \frac{|Q|^{2}}{r} \mathrm{~d} r\right\|_{L_{r}^{p /(p-2)}}+\left\|Q \int_{0}^{r} \Im\left(\bar{Q} Q_{r}\right) \mathrm{d} r\right\|_{L_{r}^{p /(p-2)}} \lesssim\|Q\|_{L_{r}^{2 p /(p-2)}}^{2}\|Q\|_{L_{r}^{p}}
$$

From (41) and (42), the norm of $M$ is controlled by

$$
\|M\|_{L_{\mathbb{R}^{3}}^{p}} \lesssim\left\|Q_{0}\right\|_{L_{r}^{2}}+\|Q\|_{L_{r}^{2 p /(p-2)}}^{2}\|Q\|_{L_{r}^{p}}
$$

Equation (39) also shows the equivalence relations of the two norms to hold as follows:

$$
\left\|M_{0}\right\|_{L_{\mathbb{R}^{3}}^{2}} \sim\left\|Q_{0}\right\|_{L_{r}^{2}} \quad \text { and } \quad\|M\|_{L_{\mathbb{R}^{3}}^{p}}^{3} \sim\|Q\|_{L_{r}^{p}}^{3}
$$

Hence, (43) implies that

$$
\|M\|_{L_{\mathbb{R}^{3}}^{p}} \lesssim\left\|M_{0}\right\|_{L_{\mathbb{R}^{3}}^{2}}+\|M\|_{L_{r}^{2 p /(p-2)}}^{2}\|M\|_{L_{r}^{p}}
$$

If $\left\|M_{0}\right\|_{L_{\mathbb{R}^{3}}^{2}}$ (or $\left\|Q_{0}\right\|_{L_{r}^{2}}$ ) is small enough, (44) and $L^{2}\left(\mathbb{R}^{3}, \mu\right) \subset L^{2 p /(p-2)}\left(\mathbb{R}^{3}, \mu\right)$ (where we have the measure $\mu$ on $\mathbb{R}^{3}$ ) indicate a global in time estimate

$$
\|M\|_{L_{\mathbb{R}^{3}}^{p}} \lesssim\left\|M_{0}\right\|_{L_{\mathbb{R}^{3}}^{2}}
$$

which implies

$$
\left\|\partial_{r} S\right\|_{L_{\mathbb{R}^{3}}^{p}} \lesssim\left\|\partial_{r} S_{0}\right\|_{L_{\mathbb{R}^{3}}^{2}}
$$

Differentiating (with respect to $x_{j}, j=1,2,3$ ) (25) we get

$$
i M_{x_{j} t}-(i \beta-\alpha) \Delta M_{x_{j}}=\widetilde{H}_{3}
$$


Zhong et al. Boundary Value Problems

(2020) 2020:96

Page 15 of 18

where

$$
\begin{aligned}
\left|\widetilde{H}_{3}\right| \leq & C_{1}\left(\frac{|M||Q|^{2}}{r}+|M| \Re\left(Q_{x_{i}} \bar{Q}\right)\right) \\
& +C_{2}\left|M_{x_{j}}\right|\left(|Q|^{2}+\int_{0}^{r} \Im\left(\bar{Q} Q_{r}\right) \mathrm{d} r+\int_{0}^{r} \frac{|Q|^{2}}{r^{\prime}} \mathrm{d} r^{\prime}\right) \\
= & C_{1} R_{1}+C_{2} R_{2},
\end{aligned}
$$

where $C_{1}$ and $C_{2}$ are constants.

Lemma 2.5 also indicates a bounded

$$
\left\|M_{x_{j}}\right\|_{L^{\infty} L_{\mathbb{R}^{3}}^{2}}+\left\|M_{x_{j}}\right\|_{L_{\mathbb{R}^{3}}^{p}} \lesssim\left\|M_{x_{j} 0}\right\|_{L_{\mathbb{R}^{3}}^{2}}+\left\|\widetilde{H_{3}}\right\|_{L_{\mathbb{R}^{3}}^{p /(p-1)}},
$$

where $M_{x_{j}}$ is the initial value of $M_{x_{j}}$.

To obtain the $L_{\mathbb{R}^{3}}^{p /(p-1)}$ estimate for $\widetilde{H}_{3}$, we need to estimate the norm for $R_{1}$ :

$$
\begin{aligned}
\left\|R_{1}\right\|_{L_{\mathbb{R}^{3}}^{p /(p-1)}} & \leq\left\|\frac{|Q|^{3}}{r}+Q_{x_{i}}|Q|^{2}\right\|_{L_{\mathbb{R}^{3}}^{p /(p-1)}} \\
& \leq\left\|Q Q_{x_{i}}|Q|^{2}\right\|_{L_{\mathbb{R}^{3}}^{p /(p-1)}}+\left\|\frac{|Q|^{3}}{r}\right\|_{L_{\mathbb{R}^{3}}^{p /(p-1)}} \\
& \lesssim\left\|Q_{x_{i}}\right\|_{L_{\mathbb{R}^{3}}^{p}}\|Q\|_{L_{\mathbb{R}^{3}}^{2 p /(p-1)}}^{2}+\|Q\|_{L_{\mathbb{R}^{3}}^{2 p /(p-1)}}^{2}\left\|\frac{Q}{r}\right\|_{L_{\mathbb{R}^{3}}^{p}} \\
& \lesssim\|Q\|_{L_{\mathbb{R}^{3}}^{2 p /(p-1)}}^{2}\left\|Q_{x_{i}}\right\|_{L_{\mathbb{R}^{3}}^{p}} \\
& \lesssim\|Q\|_{L_{r}^{2 p /(p-1)}}^{2}\left\|Q_{x_{i}}\right\|_{L_{\mathbb{R}^{3}}^{p}} .
\end{aligned}
$$

By this inequality and the Hardy inequality, the space norm of $R_{2}$ can be bounded by

$$
\begin{aligned}
& \left\|R_{2}\right\|_{L_{\mathbb{R}^{3}}^{p /(p-1)}} \\
& \lesssim\left\|\left|M_{x_{j}}\left\|\left.Q\right|^{2}\right\|_{L_{\mathbb{R}^{3}}^{p /(p-1)}}+\left\|\left|M_{x_{j}}\right| \int_{0}^{r} \frac{|Q|^{2}}{r^{\prime}} \mathrm{d} r^{\prime}\right\|_{L_{\mathbb{R}^{3}}^{p /(p-1)}}+\left\|\left|M_{x_{j}}\right| \int_{0}^{r} \Im\left(\bar{Q} Q_{r}\right) \mathrm{d} r\right\|_{L_{\mathbb{R}^{3}}^{p /(p-1)}}\right.\right. \\
& \lesssim\left\|M_{x_{j}}\right\|_{L_{\mathbb{R}^{3}}^{p}}\|Q\|_{L_{\mathbb{R}^{3}}^{2 p /(p-1)}}^{2}+\left\|M_{x_{j}}\right\|_{L_{\mathbb{R}^{3}}^{p}}\left\|\int_{0}^{r} \frac{|Q|^{2}}{r^{\prime}} \mathrm{d} r^{\prime}\right\|_{L_{\mathbb{R}^{3}}^{p /(p-1)}} \\
& \quad+\left\|M_{x_{j}}\right\|_{L_{\mathbb{R}^{3}}^{p}}\left\|\int_{0}^{r} \Im\left(\bar{Q} Q_{r}\right) \mathrm{d} r\right\|_{L_{\mathbb{R}^{3}}^{p /(p-1)}} \\
& \lesssim\left\|M_{x_{j}}\right\|_{L_{\mathbb{R}^{3}}^{p}}\|Q\|_{L_{\mathbb{R}^{3}}^{2 p /(p-1)}}^{2}+\left\|M_{x_{j}}\right\|_{L_{\mathbb{R}^{3}}^{p}}\|Q\|_{L_{\mathbb{R}^{3}}^{2 p /(p-1)}}^{2}+\left\|M_{x_{j}}\right\|_{L_{\mathbb{R}^{3}}^{p}}\left\||Q|^{2}\right\|_{L_{\mathbb{R}^{3}}^{p /(p-1)}} \\
& \lesssim\|Q\|_{L_{r}^{2 p /(p-1)}}^{2}\left\|M_{x_{i}}\right\|_{L_{\mathbb{R}^{3}}^{p}} \cdot
\end{aligned}
$$


According to the estimates (45)-(47), we obtain

$$
\begin{aligned}
& \left\|M_{x_{i}}\right\|_{L^{\infty} L_{\mathbb{R}^{3}}^{2}}+\left\|M_{x_{i}}\right\|_{L_{\mathbb{R}^{3}}^{p}} \\
& \quad \lesssim\left\|M_{x_{j} 0}\right\|_{L_{\mathbb{R}^{3}}^{2}}+\|Q\|_{L_{r}^{2 p /(p-1)}}^{2}\left\|Q_{x_{i}}\right\|_{L_{\mathbb{R}^{3}}^{p}}+\|Q\|_{L_{r}^{2 p /(p-1)}}^{2}\left\|M_{x_{i}}\right\|_{L_{\mathbb{R}^{3}}^{p}} \\
& \quad \lesssim\left\|M_{x_{j} 0}\right\|_{L_{\mathbb{R}^{3}}^{2}}+\|Q\|_{L_{r}^{2 p /(p-1)}}^{2}\left\|M_{x_{i}}\right\|_{L_{\mathbb{R}^{3}}^{p}} .
\end{aligned}
$$

If $L^{2}\left(\mathbb{R}^{3}, \mu\right) \subset L^{2 p /(p-2)}\left(\mathbb{R}^{3}, \mu\right)$, (48) means that we can find some small $\left\|Q_{0}\right\|_{L_{r}^{2}}^{2}$ and $\left\|M_{x_{j} 0}\right\|_{L_{r}^{2}}^{2}$ to control the norm

$$
\left\|M_{x_{i}}\right\|_{L^{\infty} L_{\mathbb{R}^{3}}^{2}}+\left\|M_{x_{i}}\right\|_{L_{\mathbb{R}^{3}}^{p}} \leq C\left(Q_{0}, M_{x_{j}}, T\right),
$$

where $C\left(Q_{0}, M_{x_{j}}, T\right)$ is a bounded function depending on the three components in the bracket.

Hence, we finish the proof of the existence theorem as follows.

Theorem 4.1 We assume the initial data $S_{0}$ satisfies $\left\|\partial_{x_{i}} \partial_{r} S_{0}\right\|_{L_{r}^{2}} \ll 1$ and $\left\|\partial_{r} S_{0}\right\|_{L_{r}^{2}} \ll 1$ $(i=1,2,3)$. Under the embedding $L^{2}\left(\mathbb{R}^{3}, \mu\right) \subset L^{2 p /(p-2)}\left(\mathbb{R}^{3}, \mu\right)\left(p \geq 2\right.$ and $\left.\frac{4 p}{3(p-2)}>2\right)$, for the radial LLG equation

$$
S_{t}-\alpha S \times\left(S_{1 r r}+\frac{2}{r} S_{1 r}\right)+\beta S \times\left[S \times\left(S_{1 r r}+\frac{2}{r} S_{1 r}\right)\right]=0,
$$

there exists a global solution

$$
S \in C\left(\mathbb{R}, H_{\mathbb{R}^{3}}^{2}\right) \cap L_{\text {loc }}^{p}\left(\mathbb{R}, W_{\mathbb{R}^{3}}^{2, p}\right) .
$$

At the same time, $\mathrm{Q}_{0} \in H_{\mathbb{R}^{3}}^{1}$ and the three-dimensional Ginzburg-Landau type equation

$$
\begin{aligned}
& i Q_{t}-(i \beta-\alpha)\left(Q_{r r}+\frac{2 Q_{r}}{r}-\frac{2 Q}{r^{2}}\right)+4 \beta Q \int_{0}^{r} \Im\left(\bar{Q} Q_{r}\right) \mathrm{d} r \\
& +2 \alpha\left(|Q|^{2} Q+4 Q \int_{0}^{r} \frac{|Q|^{2}}{r} \mathrm{~d} r\right)=0
\end{aligned}
$$

holds, and we have

$$
\mathrm{Q} \in C\left(\mathbb{R}, H_{\mathbb{R}^{3}}^{1}\right) \cap L_{\mathrm{loc}}^{p}\left(\mathbb{R}, W_{\mathbb{R}^{3}}^{1, p}\right)
$$

\subsection{Global solution in $n \geq \mathbf{4}$}

From (36), we can derive the equivalence relations of two norms as follows:

$$
\|U\|_{L_{\mathbb{R}^{n}}^{2}} \sim\|Q\|_{L_{r}^{2}}
$$

Multiplying (35) by $\bar{U}$, integrating and taking the image part, we obtain an energy equality as follows:

$$
\|U(t)\|_{L_{\mathbb{R}^{n}}^{2}}^{2}-\left\|M_{0}\right\|_{L_{\mathbb{R}^{n}}^{2}}^{2}+2 \beta \int_{\mathbb{R}^{n}}\|\nabla M(\tau)\|_{L_{\mathbb{R}^{n}}^{2}}^{2} \mathrm{~d} \tau=0,
$$


which generates an inequality on the norm of $U$ in $L_{\mathbb{R}^{n}}^{2}$ as follows:

$$
\|U(t)\|_{L_{\mathbb{R}^{n}}^{2}}^{2} \leq\left\|U_{0}\right\|_{L_{\mathbb{R}^{n}}^{2}}^{2}
$$

Similar to the proof of (44), (49) and (50) are useful to deduce the energy inequality

$$
\|U\|_{L_{\mathbb{R}^{n}}^{p}} \lesssim\left\|U_{0}\right\|_{L_{\mathbb{R}^{n}}^{2}}+\|U\|_{L_{r}^{2 p /(p-2)}}^{2}\|U\|_{L_{r}^{p}}
$$

in which the embedding $L^{2}\left(\mathbb{R}^{n}, \mu\right) \subset L^{2 p /(p-2)}\left(\mathbb{R}^{n}, \mu\right)$ and the smallness of $\left\|Q_{0}\right\|_{L_{\mathbb{R}^{n}}^{2}}$ indicate a global control as follows:

$$
\|Q\|_{L_{\mathbb{R}^{n}}^{p}} \lesssim\left\|Q_{0}\right\|_{L_{\mathbb{R}^{n}}^{2}} \quad\left(\text { or }\left\|\partial_{r} S\right\|_{L_{\mathbb{R}^{n}}^{p}} \lesssim\left\|\partial_{r} S_{0}\right\|_{L_{\mathbb{R}^{n}}^{2}}\right)
$$

Similar to the proof of (48), we have

$$
\begin{aligned}
& \left\|U_{x_{i}}\right\|_{L^{\infty} L_{\mathbb{R}^{n}}^{2}}+\left\|U_{x_{i}}\right\|_{L_{\mathbb{R}^{n}}^{p}} \\
& \quad \lesssim\left\|U_{x_{j} 0}\right\|_{L_{\mathbb{R}^{n}}^{2}}+\left\|Q_{0}\right\|_{L_{r}^{2 p /(p-1)}}^{2}\left\|U_{x_{i}}\right\|_{L_{\mathbb{R}^{n}}^{p}} \\
& \quad \lesssim\left\|U_{x_{j} 0}\right\|_{L_{\mathbb{R}^{n}}^{2}}+\left\|Q_{0}\right\|_{L_{r}^{2}}^{2}\left\|U_{x_{i}}\right\|_{L_{\mathbb{R}^{n}}^{p}} .
\end{aligned}
$$

If $\left\|Q_{0}\right\|_{L_{r}^{2}}^{2}$ and $\left\|U_{x_{j} 0}\right\|_{L_{\mathbb{R}^{n}}^{2}}$ are small enough, the energy inequalities (51) and (52) mean that there exists a global solution for the $n$-dimensional $(n \geq 4)$ LLG equation. Hence, we finished the proof of the Theorem 4.2.

Theorem 4.2 We assume $n \geq 4, p \geq 2$ and $\frac{4 p}{3(p-2)}>2$, $S_{0}$ satisfies $\left\|\partial_{x_{i}} \partial_{r} S_{0}\right\|_{L_{r}^{2}} \ll 1$ and $\left\|\partial_{r} S_{0}\right\|_{L_{r}^{2}} \ll 1(i=1,2, \ldots, n)$. If the embedding $L^{2}\left(\mathbb{R}^{3}, \mu\right) \subset L^{2 p /(p-2)}\left(\mathbb{R}^{3}, \mu\right)$ is satisfied, then, for the radial LLG equation (7), there exists a global small solution in time

$$
S \in C\left(\mathbb{R}, H_{\mathbb{R}^{n}}^{2}\right) \cap L_{\mathrm{loc}}^{p}\left(\mathbb{R}, W_{\mathbb{R}^{n}}^{2, p}\right)
$$

At the same time, $\mathrm{Q}_{0} \in H_{\mathbb{R}^{n}}^{1}$ and the n-dimensional Ginzburg-Landau type equation (23) holds,

$$
\mathrm{Q} \in C\left(\mathbb{R}, H_{\mathbb{R}^{n}}^{1}\right) \cap L_{\mathrm{loc}}^{p}\left(\mathbb{R}, W_{\mathbb{R}^{n}}^{1, p}\right)
$$

Completion of Theorem 1.1 The proof of this theorem can be obtained from Theorem 4.1 and Theorem 4.2.

\section{Acknowledgements}

The continuous support of the Guangdong University of Education is appreciated.

\section{Funding}

This work is supported by TianYuan Special Fund of the National Natural Science Foundation of China (No. 11426068), Project for Young Creative Talents of Ordinary University of Guangdong Province (No. 2014KQNCX228;

No. 2016KQNCX113), PhD Start-up Fund of Natural Science Foundation of Guangdong Province (No. 2014A030310330), Special Innovation Projects of Universities in Guangdong Province (No. 2018KTSCX161) and Fund for Science and

Technology of Guangzhou (No. 201607010352). 
Authors' contributions

All authors read and approved the final manuscript.

\section{Publisher's Note}

Springer Nature remains neutral with regard to jurisdictional claims in published maps and institutional affiliations.

Received: 2 February 2020 Accepted: 13 April 2020 Published online: 14 May 2020

\section{References}

1. Alouges, F., Soyeur, A.: On global weak solutions for Landau-Lifshitz equations: existence and nonuniqueness. Nonlinear Anal. 18, 1071-1084 (1992)

2. Bejenaru, I., lonescu, A.D., Kenig, C.E.: Global existence and uniqueness of Schrödinger maps in dimensions $d \geq 4$. Adv. Math. 215, 263-291 (2007)

3. Bejenaru, I., Ionescu, A.D., Kenig, C.E., Tataru, D.: Global Schrödinger maps in dimensions $d \geq 2$ : small data in the critical Sobolev spaces. Ann. Math. 173, 1443-1506 (2011)

4. Chang, N., Shatah, J., Uhlenbeck, K.: Schrödinger maps. Commun. Pure Appl. Math. 53(5), 590-602 (2000)

5. Ding, S., Wang, C.: Finite time singularity of the Landau-Lifshitz-Gilbert equation. Int. Math. Res. Not. 2007, Article ID rnm012 (2007). https://doi.org/10.1093/imrn/rnm012

6. Guo, B., Hong, M.: The Landau-Lifshitz equation of the ferromagnetic spin chain and harmonic maps. Calc. Var. Partial Differ. Equ. 1, 311-334 (1993)

7. Guo, B., Huang, H.: Smooth solution of the generalized system of ferro-magnetic chain. Discrete Contin. Dyn. Syst. 5, 729-740 (1999)

8. Gustafson, S., Kang, K., Tsai, T.: Schrödinger flow near harmonic maps. Commun. Pure Appl. Math. 60, 463-499 (2007)

9. Harpes, P.: Uniqueness and bubbling of the 2-dimensional Landau-Lifshitz flow. Calc. Var. Partial Differ. Equ. 20, 213-229 (2004)

10. Harpes, P.: Bubbling of approximations for the 2-D Landau-Lifshitz flow. Commun. Partial Differ. Equ. 31, 1-20 (2006)

11. Huber, A.: Periodic solutions for the Landau-Lifshitz-Gilbert equation. J. Differ. Equ. 250, 2462-2484 (2011)

12. Ionescu, A.D., Kenig, C.E.: Low-regularity Schrödinger maps, ll: global well-posedness in dimensions $d \geq 3$. Commun. Math. Phys. 271, 523-559 (2007)

13. Klainerman, S., Ponce, G.: Global small amplitude solutions to nonlinear evolution equations. Commun. Pure Appl. Math. 36, 133-141 (1983)

14. Landau, L.D., Lifshitz, E.M.: On the theory of the dispersion of magnetic permeability in ferromagnetic bodies. Phys. Z. Sowjetunion 8, 153-169 (1935). Reproduced in: Collected Papers of L. D. Landau, pp. 101-114. Pergamon, New York (1965)

15. Lin, J., Lai, B., Wang, C.: Global well-posedness of the Landau-Lifshitz-Gillbert equation for initial data in Morrey spaces. Calc. Var. Partial Differ. Equ. 54, 665-692 (2015)

16. Melcher, C.: Existence of partially regular solutions for Landau-Lifshitz equations in $R^{3}$. Commun. Partial Differ. Equ. 30, 567-587 (2005)

17. Melcher, C.: Global solvability of the Cauchy problem for the Landau-Lifshitz-Gilbert equation in higher dimensions. Indiana Univ. Math. J. 61, 1175-1200 (2012)

18. Merle, F., Raphaël, P., Radnianski, I.: Blowup dynamics for smooth data equivariant solutions to the critical Schrödinger map problem. Invent. Math. 193, 249-365 (2013)

19. Perelman, G.: Blow up dynamics for equivariant critical Schrödinger maps. Commun. Math. Phys. 330, 69-105 (2014)

20. Shatah, J.: Global existence of small solutions to nonlinear evolution equations. J. Differ. Equ. 46, 409-423 (1982)

21. Sulem, P.L., Sulem, C., Bardos, C.: On the continuous limit for a system of classical spins. Commun. Math. Phys. 107, 431-454 (1986)

22. Zhong, P., Wang, S., Zeng, M.: Two blowup solutions for inhomogeneous isotropic Landau-Lifshitz equation. J. Math. Anal. Appl. 409, 74-83 (2014)

23. Zhong, P., Yang, G.: Finite time blowup of multidimensional inhomogeneous isotropic Landau-Lifshitz equation on a hyperbolic space. Comput. Math. Appl. 73, 433-449 (2017)

24. Zhong, P., Yang, G., Ma, X.: Global existence of Landau-Lifshitz-Gilbert equation and self-similar blowup of harmonic map heat flow on $S^{2}$. Math. Comput. Simul. 174, 1-18 (2020)

\section{Submit your manuscript to a SpringerOpen ${ }^{\circ}$ journal and benefit from:}

- Convenient online submission

- Rigorous peer review

- Open access: articles freely available online

- High visibility within the field

- Retaining the copyright to your article

Submit your next manuscript at $\boldsymbol{~ s p r i n g e r o p e n . c o m ~}$ 